\title{
Controlling the Phase Front of Optical Fiber Beams using High Contrast Metastructures
}

\author{
Amir Arbabi ${ }^{1}$, Mahmood Bagheri ${ }^{2}$, Alexander J. Ball ${ }^{1}$, Yu Horie ${ }^{1}$, David Fattal ${ }^{3}$, Andrei Faraon ${ }^{1}$ \\ ${ }^{I}$ T. J. Watson Laboratory of Applied Physics, California Institute of Technology, 1200 E California Blvd., Pasadena, CA 91125 \\ ${ }^{2}$ Jet Propulsion Laboratory, California Institute of Technology, Pasadena, CA 91109, USA \\ ${ }^{3}$ Hewlett-Packard Laboratories, 1501 Page Mill Road, Palo Alto, CA 94304, USA \\ amir@caltech.edu
}

\begin{abstract}
The phase of optical beams can be modified by high contrast sub-wavelength periodic structures with gradually varying geometrical features. We present design, simulation, fabrication and characterization of planar micro-lenses shaping the beam of optical fibers.

OCIS codes: (050.6624) Subwavelength structures; (050.1965) Diffractive lenses; (050.0050) Diffraction and gratings
\end{abstract}

Planar optical elements based on sub-wavelength structures enable excellent control of the phase and amplitude of optical fields. These structures consist of a two-dimensional pattern created by locally and gradually modifying the parameters of a periodic structure. The patterns are etched directly in a thin layer of material with a high index of refraction surrounded by lower refractive index materials. The unprecedented phase control provided by these nanopatterned metastructures allows for simple implementation of arbitrary transmissive and reflective phase masks. Planar lenses [1,2], and focusing mirrors [3.4] have been simulated and implemented using this technique. These structures are fabricated using standard micro-fabrication techniques which allow for mass production of these devices using a parallel fabrication process. These planar devices have the potential to replace conventional bulky optical components used in free-space optics in some specific applications. As we show here, one of the promising applications for these devices is the modification of the output beam of optical fibers. In particular, we show that the light emitted out of a cleaved fiber can be collimated using a planar lens. Such a lens has the potential to be mounted directly on the tip of optical fibers. The degrees of freedom in the design of these lenses can enable formation of arbitrary beam profiles, and may be used to match the mode of an optical fiber to that of an integrated optical waveguide to increase the coupling efficiency to photonic chips.

These planar micro-lens structures are quasi-periodic circular Silicon posts in a hexagonal lattice sitting on a $3 \mu \mathrm{m}$ thick silicon dioxide membrane. The amplitude and phase of the transmission coefficient for a plane wave impinging normal to the membrane was calculated using the rigorous coupled mode analysis (RCWA) technique for a series of structures with different lattice constants, post radii, and post heights. The simulations were performed at the wavelength of $\lambda=1550 \mathrm{~nm}$ and the lattice constant of the structure was kept sub-wavelength to prevent propagation of the higher orders of diffraction. For a periodic structure with the lattice constant of $0.76 \mu \mathrm{m}$ and the posts' height of $1 \mu \mathrm{m}$, we achieved total control on the phase of the transmitted wave in the 0 to $2 \pi$ range by just varying the posts' radii while keeping the transmission relatively large and constant. The transmission amplitude and phase, and reflection as a function of the duty cycle, which is defined as the ratio of the post diameters to the lattice constant, for this periodic structure are shown in Fig. 1(a). We used this periodic structure to implement arbitrary phase masks by selecting post radii at each lattice site corresponding to the given value of phase at that site. In particular, we designed a phase mask for a fiber collimator with working distance of $400 \mu \mathrm{m}$ such that the phase front of the input beam is flattened when passing through the phase mask. The phase mask sampled at the hexagonal lattice sites is shown in Fig. 1(b). The designed micro-lens performance was confirmed using the finite difference time domain (FDTD) technique. Here, the light from the cleaved fiber facet was first propagated from the fiber to the vicinity of the microlens using the plane wave expansion method. Then, the fiber's light at the vicinity of the micro-lens was used as an excitation source for the FDTD simulation of the micro-lens. The color coded amplitude of the dominant component of the electric field in the FDTD simulation is shown in the top part of Fig. 1(c). Using FDTD simulation we found the light transmitted through the micro-lens, and propagated it away from the lens using the plane wave expansion method. The result is shown in the lower part of Fig. 1(c), and confirms the collimation of the beam by the micro-lens.

The flat micro-lenses are fabricated on a silicon on insulator substrate with silicon and buried oxide thicknesses of $1 \mu \mathrm{m}$ and $3 \mu \mathrm{m}$, respectively. The designed pattern was defined on a positive resist using e-beam lithography. Then a $70 \mathrm{~nm}$ aluminum oxide layer was deposited by e-beam evaporation and patterned using the lift-off process. The patterned aluminum oxide was then used as a dry etch mask for etching silicon and forming the posts. Figures 1(d) and (e) show scanning electron microscope (SEM) images of a fabricated micro-lens and zoomed in view of the silicon posts. 

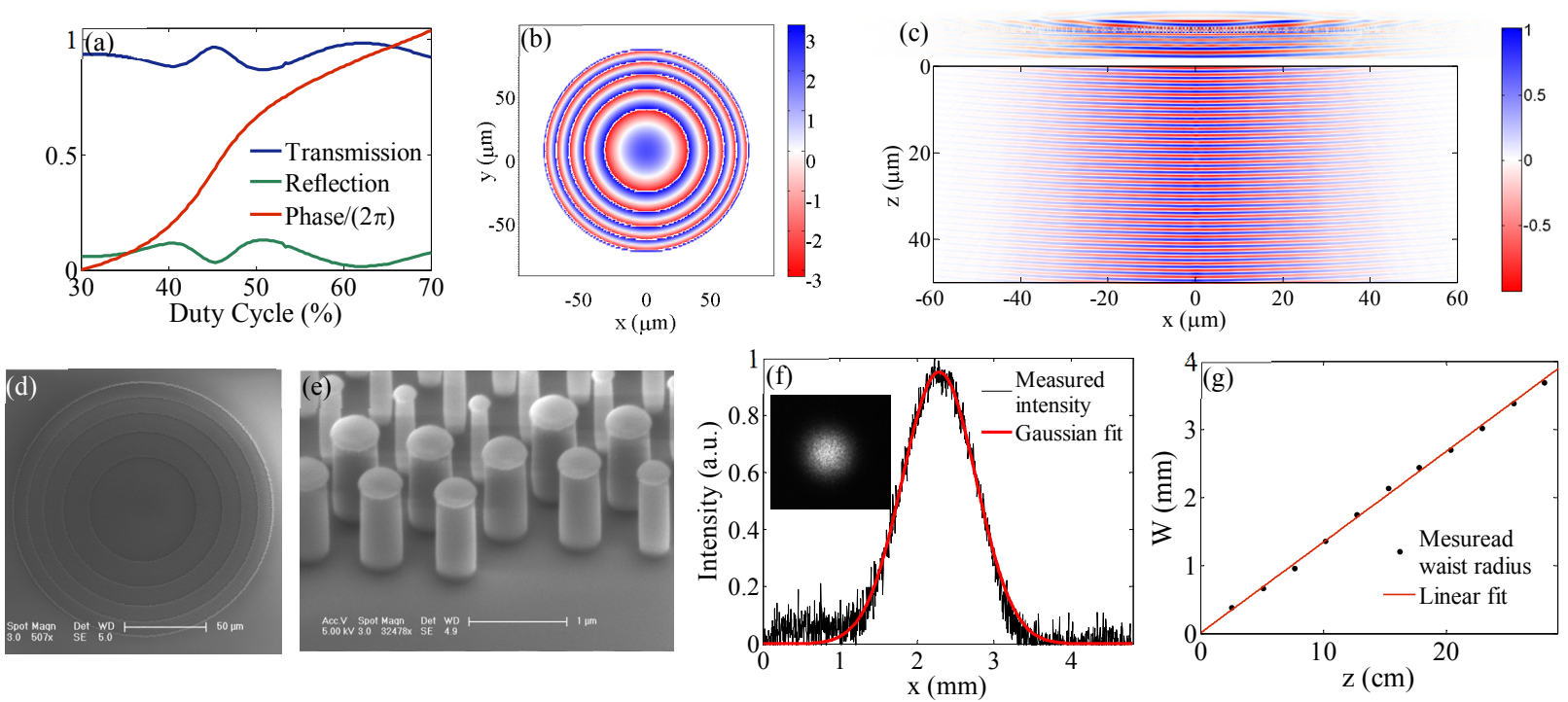

Fig. 1. (a) Transmission phase and amplitude and reflection amplitude of a 2D periodic array of silicon posts in a hexagonal lattice. (b) Phase mask of a fiber collimator with working distance of $400 \mu \mathrm{m}$ sampled at the hexagonal lattice sites. (c) Amplitude of the dominant component of the electric field close to the micro-lens (top part) and further away from the lens (bottom part). (d) SEM image of the fabricated micro-lens, and (e) close view of the etched silicon posts. (f) Intensity profile of the beam transmitted through the micro-lens along the $\mathrm{x}$ direction measured $7.6 \mathrm{~cm}$ away from the lens. A Gaussian fit to the measured intensity profile is also shown. The inset shows the 2D image recorded by the near IR camera. (g) The beam radius measured at different distances from the lens and a linear fit to the data.

The silicon oxide membranes were formed by removing the silicon substrate underneath the micro-lenses. The backside windows were defined using an aluminum oxide lift-off process similar to the one used for the top side pattern. Then, the substrate was completely etched inside the windows using the standard silicon Bosch process followed by a $\mathrm{KOH}$ wet etch step. The backside wet etch was necessary for stopping the etch exactly at the buried oxide layer, and to prevent roughening the back surface. In the final device the silicon posts are resting on the buried oxide membrane.

The collimating flat micro-lenses were characterized by illuminating them with the light from a cleaved single mode fiber. The fiber was inserted through the windows opened on the backside of the substrate. The transmitted light through the micro-lens was measured using a photodetector and a near IR camera. The distance between the fiber tip and the micro-lens was adjusted for achieving a collimated beam. The transmission of a micro-lens was measured as $86 \pm 1 \%$ over the entire tunability range $(1450-1580 \mathrm{~nm})$ of our laser. The intensity profile of the transmitted beam at several different distances from the micro-lens was recorded with a near IR camera with phosphorous coating. Figure 1(f) shows the intensity profile measured $7.6 \mathrm{~cm}$ away from a micro-lens. The intensity profile along the $\mathrm{x}$ direction is shown in this figure along with its Gaussian fit. Inset of Fig. 1(f) shows the 2D intensity profile. The noise in the measured intensities is caused by the phosphorous coating on the camera. Beam radii at different distances from the micro-lens were found by fitting Gaussian functions to the intensity profiles measured at these distances, and are plotted in Fig. 1(g). A line is fitted to the measured data and the divergence angle of $1.44^{\circ}$ was calculated using its slope. From the divergence angle and the expected beam radius of $\mathrm{W}=35.2 \mu \mathrm{m}$ of the fiber light $400 \mu \mathrm{m}$ away from its cleaved tip, we find beam quality factor of $\mathrm{M}^{2}=1.05$. The good quality of the collimated beam and its reasonable transmission through the micro-lens demonstrate the potential of these planar devices for finding practical applications. In particular, because of the freedom in choosing the desired phase mask, any arbitrary wavefronts can be realized.

\section{References}

[1] D. Fattal, J. Li, Z. Peng, M. Fiorentino, and R. G. Beausoleil, "Flat dielectric grating reflectors with focusing abilities," Nature Photon. 4, 466-470 (2010).

[2] D. Fattal, J. Li, Z. Peng, M. Fiorentino, and R. G. Beausoleil, "A Silicon Lens for Integrated Free-Space Optics," in Advanced Photonics, OSA Technical Digest (CD) (Optical Society of America, 2011), paper ITuD2.

[3] F. Lu, F. G. Sedgwick, V. Karagodsky, C. Chase, and C. J. Chang-Hasnain, "Planar high-numerical-aperture low-loss focusing reflectors and lenses using subwavelength high contrast gratings," Opt. Express 18, 12,606-12,614 (2010).

[4] Annett B. Klemm, Daan Stellinga, Emiliano R. Martins, Liam Lewis, Guillaume Huyet, Liam O'Faolain, and Thomas F. Krauss,

"Experimental high numerical aperture focusing with high contrast gratings," Opt. Lett. 38, 3410-3413 (2013). 